Ann. Biol. anim. Bioch. Biophys., I975, 15 (4), 75'-756.

\title{
L'ÉTUDE DU CARYOTYPE BOVIN (BOS TAURUS L.) PAR LES MÉTHODES DE BANDES
}

\author{
C. P. POPESCU \\ avec la collaboration technique de Jeannine Boscher \\ U. N. C. E. I. A. et Laboratoire de Génétique factorielle, \\ Centre national de Recherches zootechniques, I. N. R. A., \\ 78350 Jouy en Josas
}

RÉSUMÉ

Les méthodes des bandes $Q, G$ et $C$ ont été appliquées aux chromosomes des bovins, en vue de leur identification individuelle. Les bandes $Q$ et surtout les bandes $G$, obtenues sur des cellules aux chromosomes très despiralisés permettent l'appariement de la plupart des autosomes. Les bandes $\mathrm{C}$ ont une distribution uniforme sur tous les autosomes et de ce fait, ne facilitent pas leur identification. Néanmoins, leur application sur des cellules porteuses de la fusion de type I/29 ont montré un chromosome fusionné monocentrique, ce qui pourrait indiquer une origine ancienne. L'amélioration de la technique de coloration de l' $\mathrm{HC}$ a permis par ailleurs, la mise en évidence d'un polymorphisme quantitatif individuel, pour les deux premières paires autosomales.

L'introduction par CASPERSon et al. (I970) de la microscopie en fluorescence, après coloration à la quinacrine, dans l'étude des chromosomes a ouvert une nouvelle voie à la cytogénétique humaine et animale. Depuis, plusieurs techniques nouvelles ont été mises au point, qui produisent des bandes spécifiques sur chaque paire chromosomique, les rendant ainsi identifiables. Une standardisation de 1'idiogramme humain a été réalisée pour chaque type de bandes (PARIS CONFÉRENCE, I97I) ce qui a permis d'établir une nomenclature cohérente et généralement adoptée en cytogénétique humaine. La finesse de l'analyse effectuée à l'aide de ces méthodes a permis la découverte de nombreuses anomalies qui avant échappaient à l'observation, par les techniques courantes.

I a plupart des techniques de bandes ont été adaptées aux animaux de laboratoire et ensuite aux animaux domestiques. Toutefois, à l'exception de la souris, pour 
aucune espèce domestique il n'existe actuellement un idiogramme standard " à bandes $\%$.

Chez les bovins (Bos taurus L.) sur l'ensemble d'anomalies de nombre et de structure connues actuellement, deux seulement ont été localisées avec précision : les fusions centriques $\mathrm{r} / 29$ (Gustavsson, communication personnelle) et 2/4 (PoLLock, I974). Cette situation est due à l'absence d'un idiogramme standard à bandes et aux difficultés propres à cette espèce. Fin effet, les chromosomes du bœuf domestique sont particulièrement difficiles à étudier en raison de leur nombre très élevé, 60, et surtout de leur forme puisque les autosomes sons tous acrocentriques.

Nous présentons dans cette note le résultat de l'application de certaines techniques de bandes sur le caryotype des bovins appartenant à plusieurs races françaises.

\section{MA'TÉRIEL ET MÉTHODES}

L'étude a été effectuée simultanément avec une recherche sur les anomalies chromosomiques parmi les animaux utilisés dans les centres d'insémination artificielle. Des préparations chromosomiques particulièrement réussies, appartenant à izo animaux de races différentes ont été traités par les méthodes de bandes $Q, G$ et $C$.

Les cultures cellulaires ont été effectuées selon la méthode de Grouchy et al. (I965). Une partie des lames ainsi obtenues, séchées à la flamme et colorées au Giemsa, servait à l'étude du caryotype de l'animal. Une autre série de lames, séchées à l'air, servait aux techniques de bandes. Parfois, ces dernières provenaient des cultures cellulaires parallèles, réalisées spécialement sans traitement à la colchicine.

Les bandes $Q$. Les lames ont été, après passage dans une série d'alcools, colorés à la quinacrine moutarde (Sigma) et montées dans une solution tampon au $\mathrm{pH} 6,8$. Les observations ont été faites avec un microscope Leitz-Ortholux équipé d'un illuminateur Ploëm avec une lampe HBO 200 , un filtre d'excitation BGi2, deux filtres interférentiels KP49o et un filtre d'arrêt K-51o. Les photographies ont été prises avec un Leitz-Orthomat sur un film Kodak Tri X.

Les bandes $G$. Les bandes $G$ ont été obtenues par le traitement à la trypsine selon Ia méthode de SEABright (I97I) ou par la méthode dite ASG de Evans et al. (I97I), légèrement modifiée.

Les bandes $C$. La coloration spécifique de l'hétérochromatine a été obtenue dans un premier temps par la méthode de ARRIGHI et Hsu (I97I) sur un petit nombre de lames. Cette méthode a deux inconvénients majeurs : la durée du traitement extrêmement longue (24 h) et la mauvaise conservation de la structure chromosomique. C'est pourquoi nous avons adapté la méthode de Summer qui, après de légères modifications, donne de bons résultats et permet une application routinière sur un grand nombre d'animaux. Telle que nous l'utilisons actuellement elle consiste en un traitement de $5 \mathrm{mn}$, dans une solution $5 \mathrm{p}$. Ioo d'hydroxide de baryum à $5^{\circ} \mathrm{C}$, suivi d'une incubation de deux heures dans une solution $2 \times \mathrm{SSC}$ à $65^{\circ} \mathrm{C}$.

Les bandes $\mathrm{C}$ ont été également observées en fluorescence, après coloration à l'acridine orange.

\section{RÉSULTATS}

\section{Les bandes $Q$ et $G$}

Ifes chromosomes bovins apparaissent, après coloration à la quinacrine moutarde, constitués d'une succession de régions sombres et de régions brillantes. Les zones centromériques et les bras courts du chromosome $\mathrm{Y}$, qui correspondent à l'em- 
placement de l'hétérochromatine constitutive, restent incolores (P1. I, fig. I). La spécificité des bandes $Q$ permet l'appariement d'un grand nombre de paires chromosomiques de cette espèce, surtout des plus grandes. Toutefois, en raison de leur taille et surtout de leur morphologie identique, les dernières paires ne peuvent pas être différenciées par cette méthode.

Le dessin des bandes est plus évident sur les métaphases traitées par la méthode des bandes $G$ aussi bien par la trypsine que par la méthode A.S.G. (P1. $2 a-c$ ). Par ces méthodes tous les chromosomes peuvent être appariés, surtout dans les cellules où ils sont très despiralisés, parce qu'ils n'ont pas subi de traitement à la colchicine. En effet, on constate que les trois dernières paires peuvent être confondues dans des cellules peu (P1. $3 a$ ) ou moyennement (P1. $3 b$ ) despiralisées, alors qu'elles deviennent distinctes sur une cellule où les chromosomes sont très despiralisés (P1. $3 c)$.

\section{Les bandes $C$}

L'hétérochromatine constitutive (HC) apparaît située sur tous les at1tosomes de Bos taurus dans la région centromérique ( $\mathrm{P} 1$. 3). Sur le chromosome Y elle est placée dans la région médiane du bras court, alors que sur l'X, elle occupe la partie proximale du bras court et la région centromérique, mais elle apparaît sur ce dernier faiblement colorée, par rapport à l'HC des autosomes.

La quantité d'HC semble égale pour les homologues de la plupart des paires autosomales. Toutefois, pour les deux premières paires, seules identifiables avec certitude par la technique courante, on trouve chez certains animaux, une différence quantitative évidente entre les deux homologues et cette différence est présente sur toutes les cellules d'un animal étudiées par cette technique (P1. 4). Sur 1'ensemble de go animaux appartenant à 5 races françaises (Normande, Limousine, Charolaise, MaineAnjou, Montbéliarde et F.F.P.N.) étudiées par cette méthode, le polymorphisme de l'HC de la première ou de la deuxième paire autosomale est présent chez II animaux appartenant à 3 races.

Les bandes $\mathrm{C}$ ont été également étudiées chez ro animaux porteurs de la fusion centrique I/29 décrits antérieurement (Popescu, I974a, b). Dans tous les cas le chromosome fusionné montre, en lumière normale après coloration au Giemsa (fig. $5 a$ ) ou en fluorescence, après coloration à l'acridine orange un seul bloc hétérochromatique sur le bras long (fig. 5 b).

\section{DISCUSSION}

Plusieurs auteurs ont déjà appliqué la méthode des bandes $Q$ et $\mathrm{G}$ aux chromosomes des bovins en vue de leur identification (HANSEN, I97I, I972; SCHNEDL, I972; Evans et coll., I973; Masuda et coll., I974; SETH et KUnZE, I974 ; SchNEdL et CzAKER, 1974). Las techniques des bandes $Q$ et $\mathrm{G}$ permettent en effet, par leur spécificité l'appariement de tous les chromosomes de cette espèce, malgré leur forme identique. Toutefois, deux chromosomes, bien qu'ils soient reconnus homologues par une des méthodes de bandes, n'acquièrent pas, pour autant, une place précise dans le caryotype. L'idiogramme de toutes les espèces dont les chromosomes ont une 
morphologie variable est basé sur deux éléments : la position du centromère et la longueur relative moyenne. Ainsi, chaque paire chromosomique occupe une place bien définie, toujours la même, dans une série décroissante, souvent divisée en plusieurs groupes. Chez Bos taurus, la position invariable du centromère chez tous les autosomes, crée des risques de confusion entre deux et même plusieurs paires voisines (POPEscu, I969). C'est pourquoi, il est important d'établir pour cette espèce, un idiogramme standard dans lequel les chromosomes soient appariés à l'aide des bandes et possèdent une place donnée, selon leur longueur moyenne établie sur un grand nombre de cellules.

Pour l'étude des anomalies impliquant en particulier les grandes paires, la méthode des bandes $Q$ pourrait être très utile. Mais pour des anomalies touchant les petites paires, la méthode des bandes $G$ serait plus indiquée puisqu'elle fournit des détails plus fins. Ainsi, le dessin obtenu pour les bandes $\mathrm{G}$ a permis l'analyse du chromosome $\mathrm{Y}$, dont la taille est comparable à celles des plus petites paires autosomales (CRIBIU et Popescu, I974).

En raison des difficultés mentionnées, dans toutes tentatives de localiser une anomalie chez cette espèce, il est nécessaire, quelle que soit la méthode de bandes utilisées, d'étudier plusieurs cellules, ayant des degrés différents de spiralisation.

Comme nous l'avons montré précédemment (Popescu, I973, 1974), les bandes C, ayant une distribution similaire sur tous les autosomes, ne facilitent pas leur identification. Au contraire, un polymorphisme individuel concernant les deux premières paires, pourrait avoir un intérêt, en tant que marqueur génétique.

Eilles servent encore à mieux comprenđre le mécanisma de formation de certaines anomalies chromosomiques, telles les fusions centriques. Ainsi, la présence d'un seul bloc d'hétérochromatine constitutive indique la perte d'un centromère et suggère une origine relativement ancienne de l'anomalie (NIEBUHR, I972 $a$, I972 $b$; EvANS et coll., I973). Deux blocs hétérochromatiques au niveau du centromère indiquerait au contraire, une origine relativement récente de l'anomalie, comme c'est le cas des métacentriques de Mus poschiavinus (FOREJT, I973) ou d'un néo-chromosome métacentrique récemment décrit chez la Chèvre (Evans et coll., 1973). Nous avons déjà remarqué l'aspect monocentrique du chromosome fusionné chez plusieurs animaux porteurs de la fusion centrique $\mathbf{I} / 29$ (PoPEscu, I973, I974). Cette observation s'avère valable pour tous les animaux étudiés, appartenant à trois races différentes. Elle serait un argument en faveur d'une origine ancienne et commune de la fusion centrique r/29 parmi les races actuelles bovines. L'attitude à adopter à l'égard des animaux porteurs d'anomalies chromosomiques de ce type serait fonction de leur mode d'apparition. Ainsi, une récurrence relativement fréquente rendrait inefficace toute mesure d'éradication.

\section{REMERCIEMENTS}




\section{SUMMARY}

\section{STUDY OF CATTIE CARYOTYPE BY BANDING METHODS}

The $Q, G$ and $\mathrm{C}$ banding methods were applied of cattle chromosomes for their individual identification. The $\mathrm{Q}$-banding and especially the G-banding pattern of the slightly contracted chromosomes permited the pairing nearly all of them.

The $\mathrm{C}$-banding are located in the centromeric regions of all autosomes and give any contribution to their identification. Therefore the $C$ pattern of the cells carrier of a $I / 29$ Robertsonian translocation showed a dicentric translocated chromosome. For this reason it is tempting to suppose an ancestral origin of this translocation.

An individual chromosome polymorphism involing constitutive heterochromatine has been found for the automosomal pairs $\mathrm{r}$ and 2 .

\section{RÉFÉRENCES BIBLIOGRAPHIQUES}

ARRIGHI F. E., HsU T. C., I97I. Localization of heterochromatin in human chromosomes. Cytogenetics, 10, 8I-86.

Caspersson T., Zech L., Johansson C., Modest E. J., i97o. Identification of human chromosomes by DNA-binding fluorescent agents. Chromosoma, 30, 2I5-227.

Cribiu E. P., Popescu C. P., I974. Un cas de chromosome Y anormalement long. Ann. Génét. Sél. anim., 6, $387-390$.

Evans H. J., Buckton K. E., Sumner A. T., I97I. Cytological mapping of human chromosomes : results obtained with quinacrine fluorescence and the acetic saline-giemsa techniques. Chromosoma, $35,310-325$.

Evans H. J., Bucklayd R. A., Sumner A. T., I973. Chromosome homology and heterochromatin in goat, sheep and ox studied by banding techniques. Chromosoma, 42, 383-402.

Forejt J., I973. Centromeric Heterochromatin Polymorphism in the House Mouse. Evidence from Inbred Strains and Natural Populations. Chromosoma, 43, I87-202.

Grovchy J. (de), Roubin P., Passage E., I964. Microtechnique pour l'étude des chromosomes humains à partir d'une culture de leucocy tes sanguins. Ann. Génét., ', 45 .

HANSEN K. M., I97I. Identification of Bovine chroniosomes by the Quinacrine Mustard Fluorescence Technique. VIth Meeting of the Scandinavian Association of Geneticists Denmark Arhus August I\%-19, I97. In Heveditas, 69.

Hansen K. M., I972. Bovine Chromosomes identified by quinacrin mustard and fluorescence microscopy. Heveditas, 70, 225-234.

MASUDA H., WAIDE Y., Hosoda T., I974. Geimsa banding patterns of bovine chromosomes. Jap. J. Zootech. Sci., 45, 424-426.

Niebuhr E., I972a. Unusual findings by fluorescence microscopy of a t (13 q 14 q). Human genetik, 15, $96-98$.

Niebuhr E., I972 b. Dicentric and monocentric robertsonian translocation in man. Humangenetik, 16, $217-226$.

PARIs CONFARENCE I97I. Standardization in Human Cytogenetics Birth Defects : Original Article Series, VIII, 7, I972. The National Foundation, New York.

Popescu C. P., 1969. Idiograms of Yak (Bos gmuniens) cattle (Bos taumus) and their hybrid. Ann. Génêt. Sél. anim., 1, 207-2I7.

Popescu C. P., i973. Nouvelles observations sur une fusion centrique chez Bos taurus L. Ann. Génét. Sél. anim., 5, 435-440.

Popescu C. P., I973. L'hétérochromatine constitutive dans le caryotype bovin normal et anormal. Ann. Génét., 16, I $83^{-1} 88$.

Popescu C. P., 1974, Étude du caryotype bovin par une nouvelle méthode cytogénétique : les bandes C.

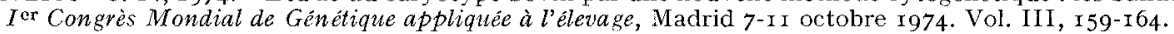

Popescu C. P., I974. Observations sur une fusion centrique chez les Bovins. (Bos taurus L.). I er Congrès Mondial de Génétique appliquée à l'élevage, Madrid, 7-II octobre I974. Vol. IIT, I65-I68.

Pollock D. L., Bowman J.-C., 1974. A robertsonian translocation in British friesian cattle. $J$. Reprod. Fert., 40, 423-432. 
SeAbright M., r97I. A rapid Banding Technique for human Chromosomes. Lancet, ii, 971-972.

Seth P. K., Kunze W. P., I974. Differential chromosomal staining in Bos taurus (Cattle). Cytologia, $39,373-378$.

SCHNEDL W., r972. Giemsa banding, quinacrine fluorescence and DNA-replication in chromosomes of cattle (Bos taurus). Chromosoma, 38, 319-328.

SchNedL W., Czaker R., 1974. Centromeric heterochromatin and comparison of G-banding in cattle, goat, and sheep chromosomes (Bovidae). Cytogenet. Cell. Genet., 13, 246-255.

Sumner A. T., I972. A simple technique for demonstrating centromeric heterochromatin. Exp. Cell. Res., 75, 304-306.

\section{PLANCHE I}

\section{Bandes $Q$}

Coloration à la quinacrine 


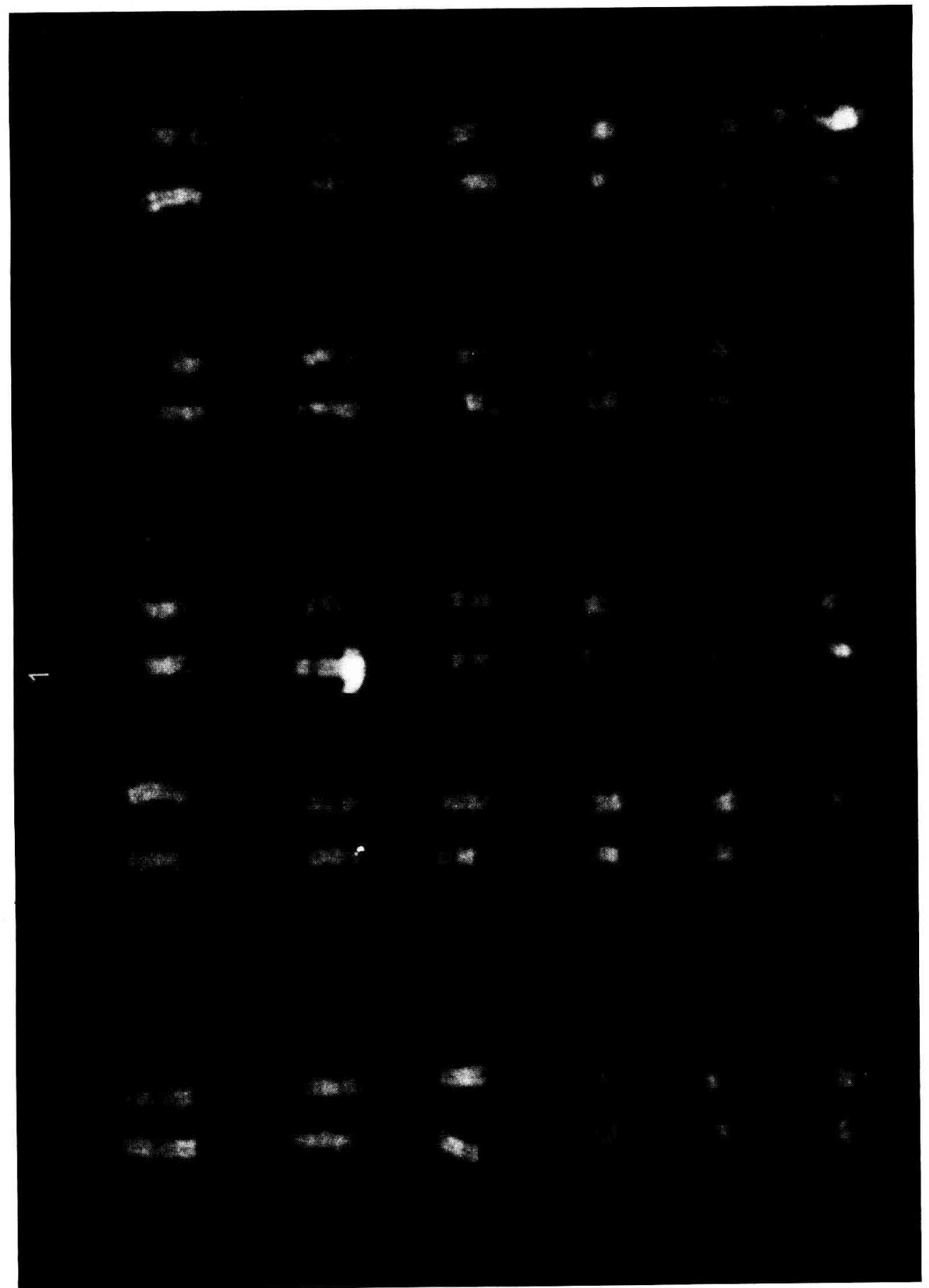

C. P. POPESCU 


\section{PLANCHE II}

Bandes G obtenues par la méthode ASG (acide-saline-giemsa). $a, b, c$ trois cellules à des degrés de déspiralisations différents avec le schéma d'analyse des bandes correspondant. 


\section{$2 a$}

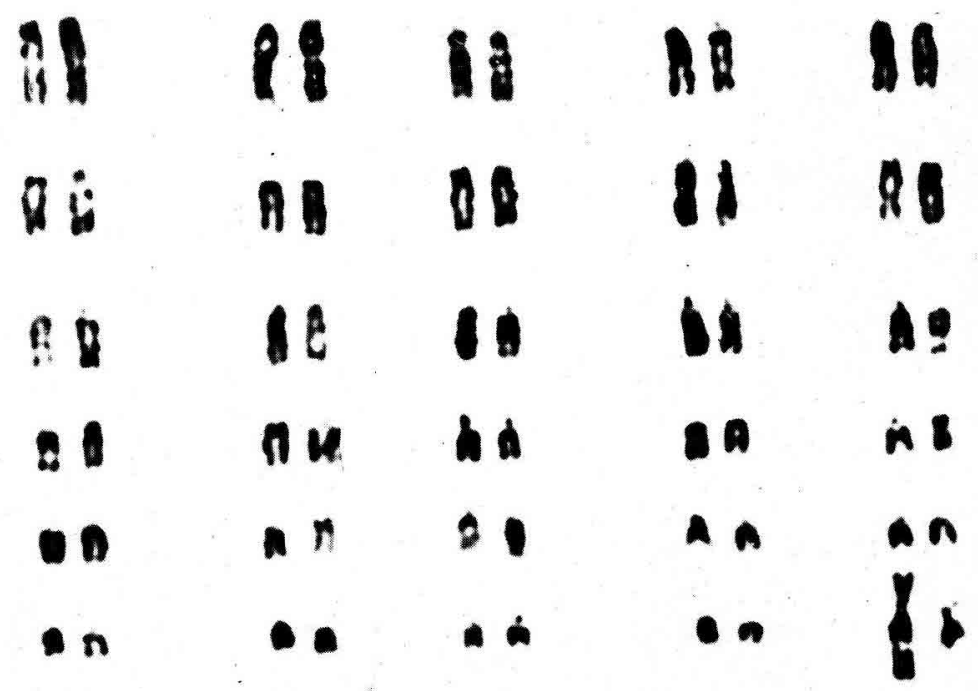
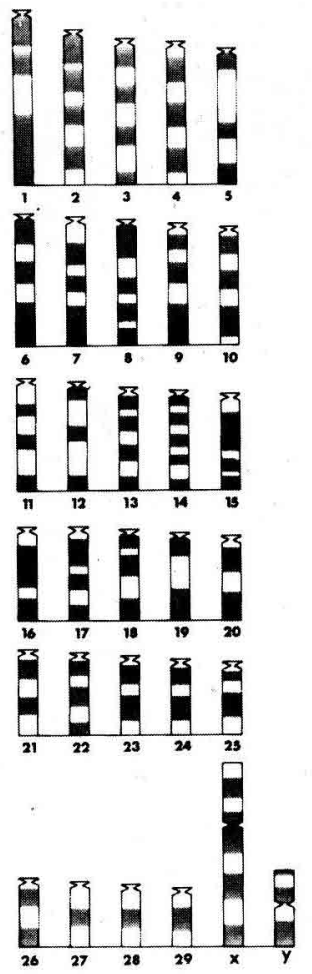

C. P. POPESCU 
$2 b$

\begin{tabular}{|c|c|c|c|}
\hline (i) & 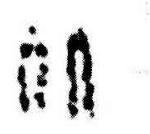 & $\| \mathfrak{H}$ & 39 \\
\hline ii & a! & $\| !$ & $\| i$ \\
\hline & $n !$ & 68 & $\because 4$ \\
\hline 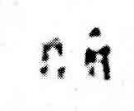 & $i n$ & 4 & $0 \pi$ \\
\hline & $0=$ & $\therefore B$ & RA \\
\hline & & 14 & in \\
\hline
\end{tabular}

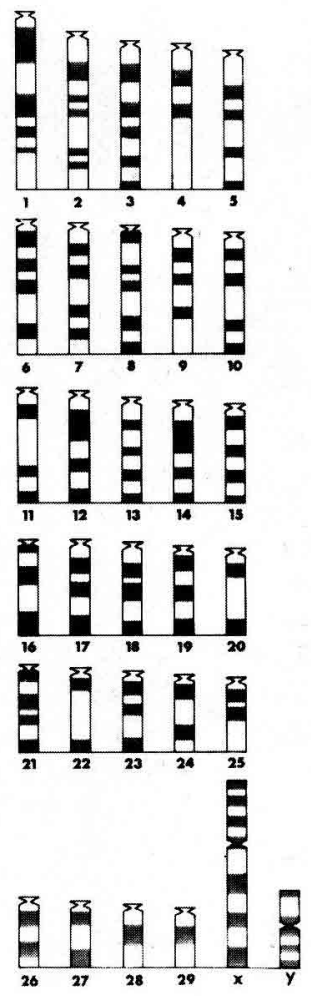


2c

8

3

83

18

$\pm 3$

1

$: 2$

18

18
18

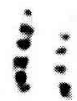

$8:$

i i

78

3

)

$1:$

1

11

r:

$\therefore$

11

is

I!

19

1

11
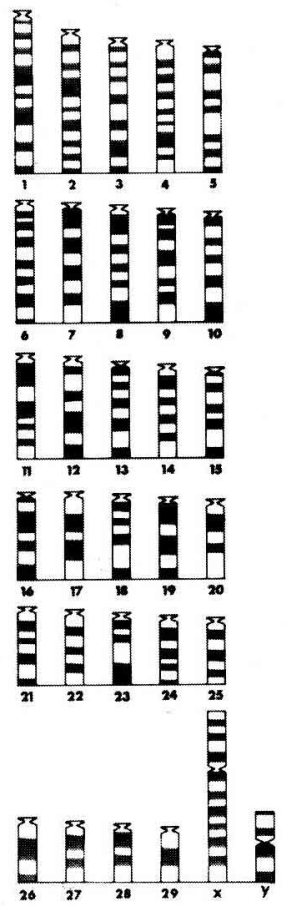
PLANCHE III

Bandes C. 

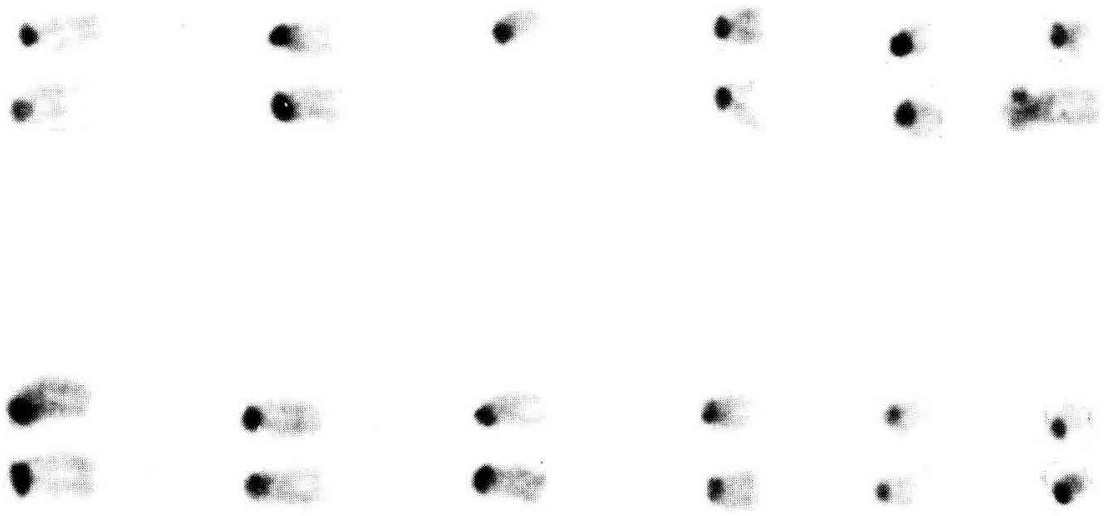

\section{$n$}
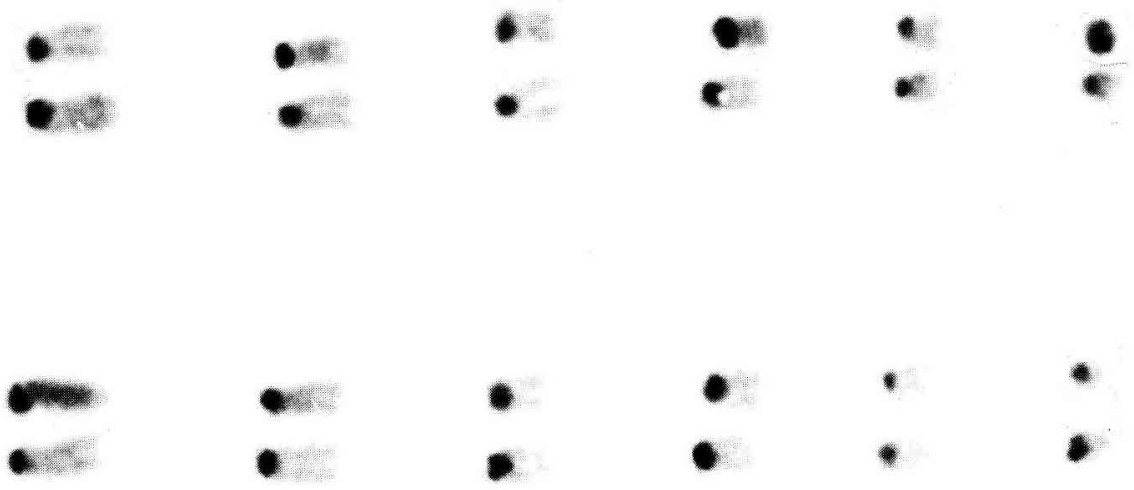

c.
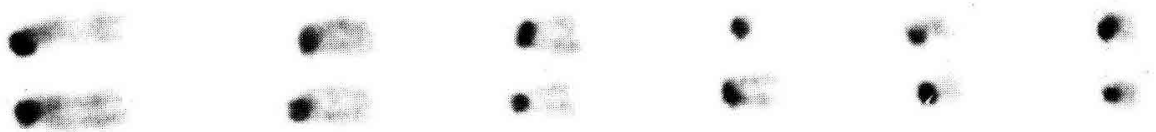

C. P. POPESCU 
PLANCHE IV

Montage des chromosomes de la première paire de 6 cellules du même animal, présentant un polymorphisme de l'HC. 


\section{4}
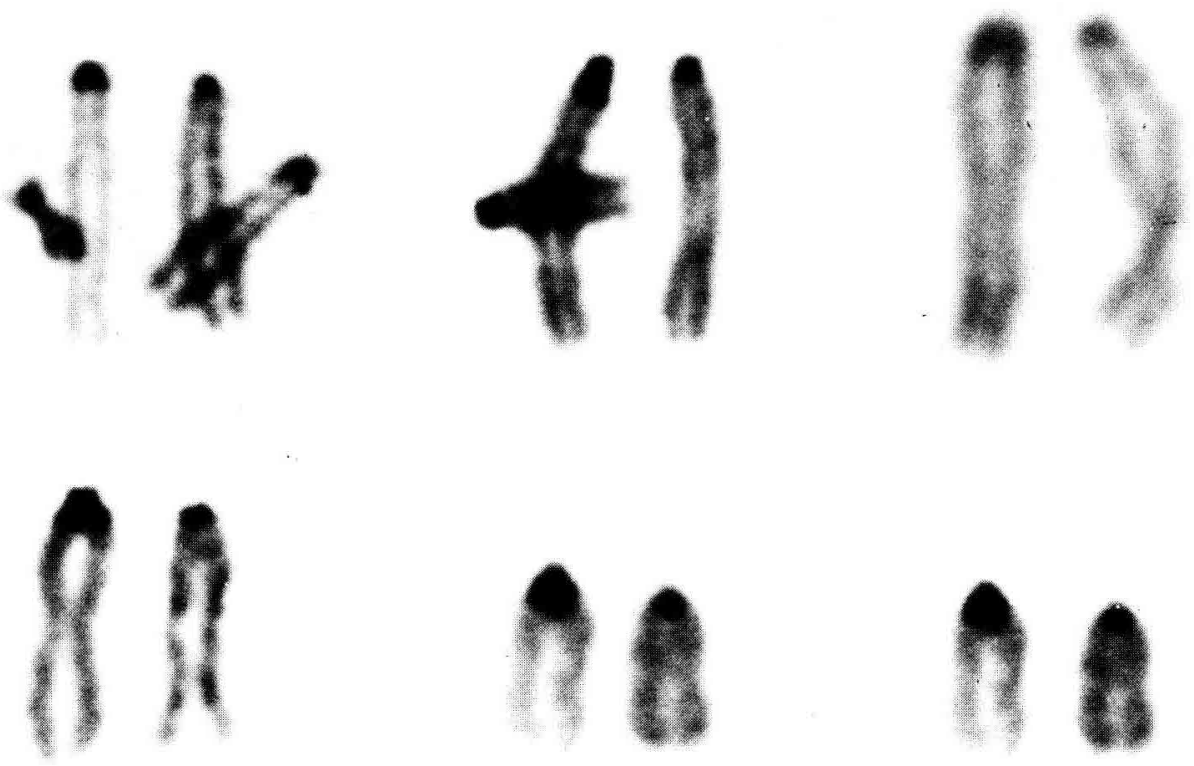

C. P. POPESCU 


\section{PLANCHE V}

Montage des chromosomes fusionnés traités pour les bandes $\mathrm{C}$ (a) et $Q$ (b). On remarque un seul bloc d'HC sur le bras long et respectivement, une région faiblement colorée correspondant à l'emplacement de l'HC. 


\section{5}
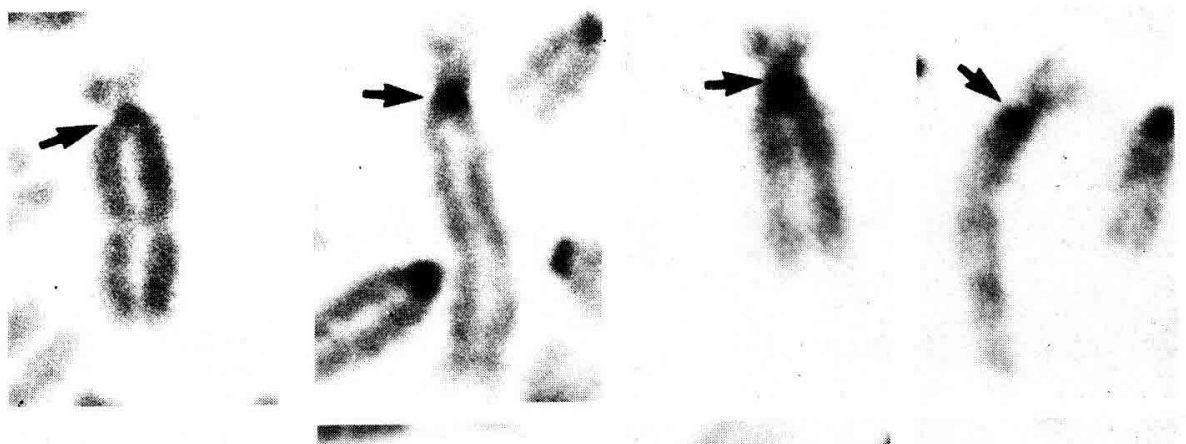

a
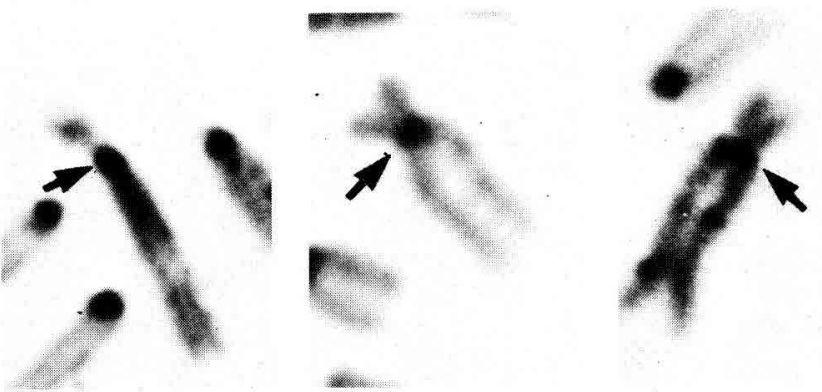

4
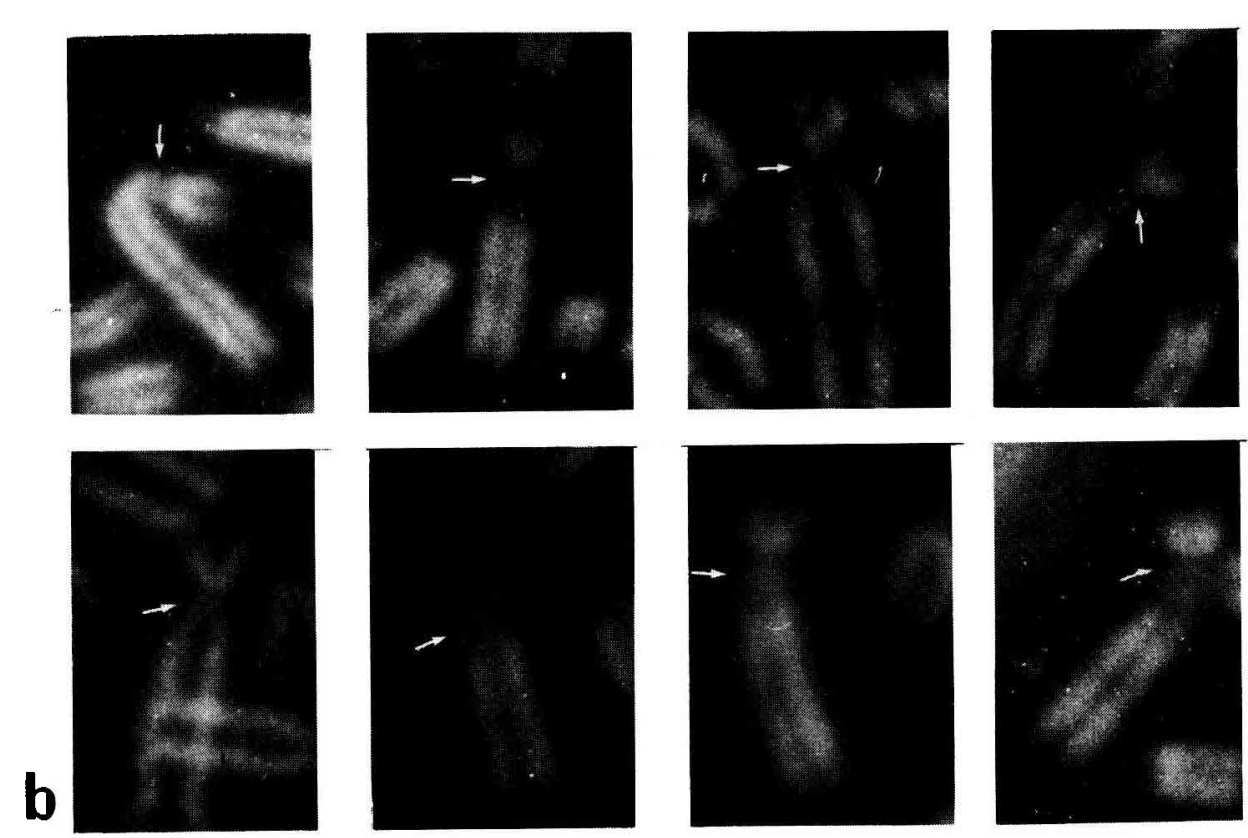

C. P. POPESCU 